\title{
DISTANCE FUNCTIONS AND THE METRIZATION PROBLEM*
}

BY A. H. FRINK

1. Introduction. The metrization problem $\dagger$ is concerned with conditions under which a topological space is metrizable, that is, is homeomorphic to a metric space. A space is metric if to every two points $a$ and $b$, a non-negative real number $a b$ is assigned satisfying the well known conditions:

I. $a b=0$ if and only if $a=b$;

II. $a b=b a$, (symmetry);

III. $a c \leqq a b+b c$, (triangle property).

A metrization theorem is usually proved by actually introducing such a distance function into the space. However, it is often easier to introduce first into a topological space a distance function satisfying the following conditions IV or V instead of III:

IV. If $a b<\epsilon$ and $c b<\epsilon$, then $a c<2 \epsilon$ (generalized triangle property);

V. For every $\epsilon>0$ there exists $\phi(\epsilon)>0$ such that if $a b<\phi(\epsilon)$ and $c b<\phi(\epsilon)$, then $a c<\epsilon$ (uniformly regular).

Condition $\mathrm{V}$ reduces to IV if $\phi(\epsilon)=\epsilon / 2$. Chittenden $\ddagger$ has shown that a space with a distance function satisfying I, II, and $\mathrm{V}$ is metrizable. Chittenden's proof is somewhat long and complicated. Furthermore, while the existence of a distance function satisfying III is proved, it is not defined directly in terms of the original distance function satisfying V. Alexandroff and Urysohn§ make use of Chittenden's theorem introducing a metric satisfying IV. Niemytski\| and W. A. Wilson T make use of Alexandroff and Urysohn's result.

Without relying on Chittenden's theorem, the present paper gives a simple, direct proof that a topological space with a distance function satisfying I, II, IV is metrizable. The method

* Presented to the Society, September 1, 1936.

$\dagger$ See Chittenden, this Bulletin, vol. 33 (1927), pp. 13-34.

$\ddagger$ Transactions of this Society, vol. 18 (1917), p. 161.

§ Comptes Rendus, vol. 177 (1923), p. 1274.

|| Transactions of this Society, vol. 29 (1927), p. 507.

T American Journal of Mathematics, vol. 53 (1931), p. 361. 
used gives a direct proof of Chittenden's theorem itself. By means of this method it is also possible to simplify greatly the proofs of several well known metrization theorems by introducing the final metric directly in terms of the original conditions. As further applications of this method of introducing a triangle axiom metric, some new metrization theorems are proved for spaces with unsymmetric distance functions and in terms of the neighborhoods of spaces satisfying Hausdorff's first countability condition.

2. Condition IV and Chittenden's Theorem. Lemma. If $a, x_{1}$, $x_{2}, \cdots, x_{n}, b$ are any $n+2$ points of a space with a distance function satisfying I, II, and IV, then

(1) $a b \leqq 2 a x_{1}+4 x_{1} x_{2}+4 x_{2} x_{3}+\cdots+4 x_{n-1} x_{n}+2 x_{n} b$.

Proof. Suppose the lemma false. Then there is some value of $n$ for which (1) does not hold. Let $N$ be the smallest such integer. Then

(2) $a b>2 a x_{1}+4 x_{1} x_{2}+4 x_{2} x_{3}+\cdots+4 x_{N-1} x_{N}+2 x_{N} b$,

while (1) holds for $n<N$. Now $N>1$, for with $n=1$, the relation (1) is a consequence of IV. It follows from IV that for every $x_{r}$ either

$$
a b \leqq 2 a x_{r}
$$

or

$$
a b \leqq 2 x_{r} b .
$$

If $r=1$, (3) does not hold because of (2), hence (4) does. Likewise (4) does not hold for $r=N$. Let $k$ be the largest value of $r$ for which (4) holds. Then $k<N$, and

$$
a b \leqq 2 x_{k} b .
$$

From the definition of $k$,

$$
a b \leqq 2 a x_{k+1}
$$

Since (1) holds for $n<N$,

(7) $x_{k} b \leqq 2 x_{k} x_{k+1}+4 x_{k+1} x_{k+2}+\cdots+4 x_{N-1} x_{N}+2 x_{N} b$, and 
(8) $a x_{k+1} \leqq 2 a x_{1}+4 x_{1} x_{2}+\cdots+4 x_{k-1} x_{k}+2 x_{k} x_{k+1}$.

Adding (7) and (8) and combining with (5) and (6) gives

$$
a b \leqq 2 a x_{1}+4 x_{1} x_{2}+\cdots+4 x_{N-1} x_{N}+2 x_{N} b,
$$

which contradicts (2).

In a space with a distance function satisfying I, II, and IV, this lemma makes it possible to define a new distance function satisfying III in the following simple way. Given two points $a$ and $b$, let $x_{1}, x_{2}, \cdots, x_{n}$ be any finite number of points of the space, not necessarily distinct from each other or from $a$ and $b$. Define $d(a b)$ to be the greatest lower bound of $a x_{1}+x_{1} x_{2}+\ldots$ $+x_{n-1} x_{n}+x_{n} b$ for all possible selections of $x_{1}, x_{2}, \cdots, x_{n}$ in the space. ${ }^{*}$ Then it follows from the lemma that $a b / 4 \leqq d(a b) \leqq a b$. Hence the distance function $d(a b)$ leads to the same definition of limit point as the old distance function and is equivalent to it. It clearly satisfies I, II, and III.

The same method can be used to prove that a space with a distance function satisfying I, II, and V is metrizable. Because a distance function satisfying III is necessarily continuous, while one satisfying IV or V is not, Chittenden's proof of this theorem made use of the result that a normal space has non-constant continuous functions. The following method is more direct. If the given distance function satisfies $\mathrm{V}$, it may be assumed that $\phi(\epsilon) \leqq \epsilon / 2$, since $\phi(\epsilon)$ may be replaced by any $\psi(\epsilon) \leqq \phi(\epsilon)$. Let $r_{1}=1, r_{2}=\phi\left(r_{1}\right), \cdots, r_{n+1}=\phi\left(r_{n}\right),(n=1,2, \cdots)$. Then $r_{n} \rightarrow 0$. If $a b \geqq r_{1}$, define $d(a b) \equiv 1$. If $r_{n}>a b \geqq r_{n+1}$, define $d(a b) \equiv 2^{-n}$. Then if $a b$ satisfies V, $d(a b)$ satisfies IV. Furthermore, we see that $d(a b)$ is equivalent to $a b$ since, if $a a_{n}$ approaches zero, so does $d\left(a a_{n}\right)$, and conversely. Now let $\delta(a b)$ be the greatest lower bound of $d\left(a x_{1}\right)+d\left(x_{1} x_{2}\right)+\cdots+d\left(x_{n-1} x_{n}\right)+d\left(x_{n} b\right)$ for all $x_{1}, x_{2}, \cdots, x_{n}$ of the space. Then as above, $\delta(a b)$ satisfies III and is equivalent to $a b$. This proves Chittenden's theorem that a space with a symmetric, uniformly regular distance function is homeomorphic to a metric space.

3. Conditions of Alexandroff and Urysohn, Niemytski, and

* The same method of introducing a metric has recently been used by Carrett Birkhoff, Compositio Mathematica, vol. 3 (1936), p. 429. I have learned from Chittenden that M. Aronszajn in 1933 found a different proof of a slightly weaker form of the above lemma, which has not yet been published. 
$W$. A. Wilson. The following conditions, due essentially to Alexandroff and Urysohn (loc. cit.) are necessary and sufficient that a neighborhood space (espace $V$ of Fréchet) be metrizable:

There exists in the space $H$ a sequence of families of sets $\left\{G_{1}\right\}$, $\left\{G_{2}\right\}, \cdots,\left\{G_{n}\right\}, \cdots$, the logical sum of all the sets $\left\{G_{n}\right\}$ of any one family being the entire space $H$, such that:

A. If two sets $G_{n}$ and $G_{n}{ }^{\prime}$ of the nth family $(n>1)$ have a common point, then they are both contained in some set $G_{n-1}$ of the $(n-1)$ th family.

B. If $a$ and $b$ are distinct points, there exists an $n$ such that no set $G_{n}$ of the nth family contains both $a$ and $b$.

C. Let $S_{n}(x)$ be the logical sum of all sets $G_{n}$ of the nth family which contains the point $x$. Then the sets $\left\{S_{n}(x)\right\}$ form a complete system of neighborhoods of the point $x$.

The condition corresponding to $C$ originally given by Alexandroff and Urysohn implied that the sets $\left\{G_{n}\right\}$ are all open. The form given here is more convenient for the applications to be made later in this paper to cases where the sets $\left\{G_{n}\right\}$ are not necessarily open sets.

Alexandroff and Urysohn gave the following method of introducing a metric satisfying I, II, and IV if conditions $A, B$, and $\mathrm{C}$ are satisfied. If no $G_{n}$ contains both $a$ and $b$, define $a b \equiv 1$. If $n$ is the largest integer such that $a$ and $b$ are both contained in some set $G_{n}$, define $a b \equiv 2^{-n}$. This distance satisfies IV. Alexandroff and Urysohn then used Chittenden's theorem. By combining this method with the method of the present paper, a metric satisfying I, II, and III may be introduced directly and independently of Chittenden's result as follows. For convenience let $\left\{G_{0}\right\}$ be a single set $H$, the entire space. Call a collection of sets $G_{n_{1}}, G_{n_{2}}, \cdots, G_{n_{k}}$ of the covering families a chain joining $a$ and $b$ provided $G_{n_{1}}$ contains $a$ and $G_{n_{1}}$ contains $b$, and two successive sets of the chain have a common point. Define the length of the chain to be $\sum_{r=1}^{k} 2^{-n_{r}}$, where $n_{r}$ denotes the family to which $G_{n_{r}}$ belongs. There exists at least one chain joining any two points $a$ and $b$, namely, $G_{0}$. Now define $d(a b)$ to be the greatest lower bound of the lengths of all chains joining $a$ and $b$. This distance satisfies I, II, and III, and leads to the same definition of limit point as the original neighborhoods if conditions $\mathrm{A}, \mathrm{B}$, and $\mathrm{C}$ are satisfied. This shows the 
sufficiency of Alexandroff and Urysohn's conditions. The necessity follows from the fact that in any space with a distance function satisfying I, II, and IV, the spherical neighborhoods of all points of the space with radius $2^{-n},(n=1,2, \cdots)$, form a sequence of families $\left\{G_{n}\right\}$ satisfying $\mathrm{A}, \mathrm{B}$, and $\mathrm{C}$.

Niemytski and W. A. Wilson (loc. cit.) consider the following conditions:

VIa. Given a point $a$ and a number $\epsilon>0$, there exists a number $\phi(a, \epsilon)>0$ such that if $a b<\phi(a, \epsilon)$ and $c b<\phi(a, \epsilon)$, then $a c<\epsilon$. (Called by Niemytski the local axiom of the triangle.)

VIb. If $a a_{n} \rightarrow 0$ and $b_{n} a_{n} \rightarrow 0$, then $a b_{n} \rightarrow 0$. (Coherent.)

VIc. For each point $a$ and each positive number $k$, there is a positive number $r$ such that if $b$ is a point for which $a b \geqq k$, and $c$ is any point, then $a c+b c \geqq r$. (This is Wilson's condition IV.)

Niemytski has noted that VIa and VIb are equivalent and VIc is easily seen to be equivalent to VIa. Any distance function which satisfies these conditions may be called locally regular by analogy with $\mathrm{V}$, which is called uniformly regular. By using Alexandroff and Urysohn's result and Chittenden's theorem, it has been shown that a space with a distance function satisfying I, II, and VI is metrizable.

This result can be established directly by the method of the present paper. Using VIa and assuming $\phi(a, \epsilon) \leqq \epsilon / 2$, let $\psi(a, \epsilon)$ $=\phi[a, \phi(a, \epsilon)]$. For every point $x$ of the space define $r_{1}(x) \equiv 1$, and $r_{n+1}(x) \equiv \psi\left[x, r_{n}(x)\right],(n=1,2, \cdots)$. Then $r_{n}(x) \rightarrow 0$. Let $V_{n}(x)$ be the spherical neighborhood with center $x$ and radius $r_{n}(x)$, that is, $V_{n}(x)$ consists of all points $y$ such that $x y<r_{n}(x)$. Then the sequence of families of sets $\left\{V_{1}(x)\right\},\left\{V_{2}(x)\right\}, \cdots$, where $x$ ranges over the entire space, satisfies the conditions A, B, C imposed on the sets $G_{n}$ of the modified Alexandroff and Urysohn theorem given above. Hence a metric can be introduced in exactly the same way as has been indicated for that theorem.

4. Unsymmetric Distance Functions. Most distance functions treated in the literature satisfy condition II. W. A. Wilson* has considered spaces with unsymmetric distance functions, but not in connection with the metrization problem. With an un-

* American Journal of Mathematics, vol, 53 (1931), p. 675. See also Fréchet, Les Espaces Abstraits, p. 217. 
symmetric distance function, it is necessary to be specific as to the definition of limit point in terms of distance. The definition to be used here is as follows: the point $x$ is said to be a limit point of the set $A$ if, for every $\epsilon>0$, there exists a point of the set $A$ such that $0<x a<\epsilon$. It is not necessary that $0<a x<\epsilon$. Likewise, the point $a$ is said to be the sequential limit of the sequence of points $\left\{a_{n}\right\}$ if $a a_{n} \rightarrow 0$. It is not necessary that $a_{n} a \rightarrow 0$.

Lindenbaum* has shown that condition II may be omitted from the postulates for a metric space if condition III is replaced by

IIIa. $a c \leqq a b+c b$.

The distance function is still symmetric, however, since II is a consequence of I and IIIa. Condition II may also be omitted if $I$ and IV, or I and V are assumed.

THEOREM 1. A space with an unsymmetric, uniformly regular distance function is metrizable.

Proof. Suppose $a b$ satisfies I and V. With $a=b, \mathrm{~V}$ gives the result: if $c a<\phi(\epsilon)$, then $a c<\epsilon$. Hence $a a_{n} \rightarrow 0$ if and only if $a_{n} a \rightarrow 0$. The distance function $a b$ may not be symmetric. Let $d(a b)$ be the maximum of $a b$ and $b a$. Then $d(a b)$ is symmetric and satisfies $\mathrm{V}$, and with the same function $\phi(\epsilon)$. For if $d(a b)$ $<\phi(\epsilon)$ and $d(b c)<\phi(\epsilon)$, then $a b<\phi(\epsilon)$, and $c b<\phi(\epsilon)$. Hence V implies that $a c<\epsilon$ and $c a<\epsilon$ and hence $d(a c)<\epsilon$. The distance $d(a b)$ is seen to be equivalent to $a b$. The same method may be used for condition IV, which is a special case of $\mathrm{V}$.

If the distance function is locally regular instead of uniformly regular, that is, if condition VI is satisfied instead of $\mathrm{V}$, condition II may not be omitted if the space is to be metrizable. For an example can be constructed of a space with a countable number of points and a distance function satisfying I and VI in which not every derived set is closed.

Corresponding to VI for unsymmetric distance functions, however, the following condition involving four points instead of three implies with I that the space is metrizable.

VII. Given a point a and a number $\epsilon>0$, there exists a number $\phi(a, \epsilon)>0$ such that if $a b<\phi(a, \epsilon), c b<\phi(a, \epsilon), c d<\phi(a, \epsilon)$, then $a d<\epsilon$.

\footnotetext{
* Fundamenta Mathematica, vol. 8 (1926), p. 111.
} 
THEOREM 2. A space with an unsymmetric distance function ab satisfying I and VII is homeomorphic to a metric space.

Proof. Assume $\phi(a, \epsilon) \leqq \epsilon / 2$. Let $r_{1}(a) \equiv 1, r_{2}(a) \equiv \phi\left(a, r_{1}\right)$, $\cdots, r_{n}(a) \equiv \phi\left(a, r_{n-1}\right)$. Then $r_{n}(a) \rightarrow 0$. Let $U_{n}(a)$ consist of all points $x$ such that $a x<r_{n}(a)$. Now for two points $a$ and $b$, if $U_{n}(a)$ and $U_{n}(b)$ have a point $x$ in common, $n>1$, then either $U_{n}(b) \subset U_{n-1}(a)$ or $U_{n}(a) \subset U_{n-1}(b)$. For suppose $r_{n}(a) \geqq r_{n}(b)$. Then $a x<r_{n}(a), b x<r_{n}(b) \leqq r_{n}(a)$. For any point $y$ in $U_{n}(b)$, $b y<r_{n}(b) \leqq r_{n}(a)=\phi\left(a, r_{n-1}\right)$. Hence by VII, $a y<r_{n-1}(a)$, that is, $U_{n}(b) \subset U_{n-1}(a)$. Similarly, if $r_{n}(a)>r_{n}(b), U_{n}(a) \subset U_{n-1}(b)$. Now define a distance function $d(a b)$ as follows. If $a$ and $b$ are not both in any $U_{n}(x)$, then $d(a b) \equiv 1$. If $a$ and $b$ are in $U_{n}(x)$ for some $x$ but not both in $U_{n+1}(y)$ for any $y$, define $d(a b) \equiv 2^{-n}$. For any $a$ and $b$ there will be some $n$ such that no $U_{n}(x)$ contains both $a$ and $b$, namely, an $n$ such that $2^{1-n}<\phi(a, a b)$. For if, for some $x, U_{n}(x)$ contained both $a$ and $b$, then obviously $a a<\phi(a, a b)$. Also $x a<r_{n} \leqq 2^{1-n}<\phi(a, a b), x b<r_{n}<\phi(a, a b)$. Then VII gives $a b<a b$, which is a contradiction. The distance function $d(a b)$ leads to the same definition of limit point as the distance function $a b$; that is, if $a a_{k} \rightarrow 0, d\left(a a_{k}\right) \rightarrow 0$, and conversely. For, given $a$ and $\epsilon>0$, there is an $n$ such that $r_{n-1}(a)<\epsilon$. Choose $m$ so that $2^{-m}<r_{n}(a)$. Then if $d\left(a a_{k}\right)<2^{-m}$, from the definition of $d\left(a a_{k}\right)$, there is an $x$ such that $a$ and $a_{k}$ are both in $U_{m+1}(x)$. Then $x a<r_{m+1}(x) \leqq 2^{-m}<r_{n}(a)$ and $x a_{k}<r_{m+1}(x) \leqq 2^{-m}<r_{n}(a)$. Hence, by VII, $a a_{k}<r_{n-1}(a)<\epsilon$. Conversely, for $\epsilon>0$, there is an $n$ such that $2^{-n}<\epsilon$. Now if $a a_{k}<r_{n}(a)$, then $a$ and $a_{k}$ are in $U_{n}(a)$, and hence, by the definition of $d\left(a a_{k}\right), d\left(a a_{k}\right) \leqq 2^{-n}<\epsilon$. The new distance function $d(a b)$ satisfies IV. If $d(a b)<2^{-n}$ and $d(b c)<2^{-n}$, then $a$ is in some $U_{n+1}(x)$ and $c$ in some $U_{n+1}(y)$, with the point $b$ in common. Hence both $a$ and $c$ are in either $U_{n}(x)$ or else in $U_{n}(y)$, that is, $a c \leqq 2^{-n}<2^{1-n}$. Since I and IV are satisfied, the space is metrizable.

As Fréchet has shown, ${ }^{*}$ a distance function satisfying condition I, but in general unsymmetric, can be introduced into a neighborhood space if the neighborhoods of each point $x$ form a monotonic decreasing sequence $U_{1}(x), U_{2}(x), \cdots, U_{n}(x), \cdots$, whose logical product is $x$. To do this, if $n$ is the smallest integer

* Les Espaces Abstraits, p. 217. 
such that $U_{n}(a)$ does not contain $b$, define $a b \equiv 2^{-n}$. This suggests the following metrization condition analogous to VII but stated in terms of neighborhoods.

VIII. For every point a of a neighborhood space and every integer $n$ there exists an integer $m(a, n)>n$ such that if $b$ is any point for which $U_{m}(a)$ and $U_{m}(b)$ have a point in common, then $U_{m}(b)$ c $U_{n}(a)$.

THEOREM 3. If the neighborhoods of every point $x$ of a space form a monotonic decreasing sequence $U_{1}(x), U_{2}(x), \cdots$, $U_{n}(x), \cdots$, whose logical product is $x$, and if condition VIII is satisfied, then the space is homeomorphic to a metric space.

Proof. For each point $x$ select a subsequence of its neighborhoods $\left\{U_{n}(x)\right\}$ as follows. Let $n_{1}(x)=1, n_{2}(x)=m\left[x, n_{1}(x)\right], \cdots$, $n_{r+1}(x)=m\left[x, n_{r}(x)\right]$. Let $V_{1}(x)=U_{n_{1}}(x), V_{r}(x)=U_{n_{r}}(x), \quad(r=1$, $2, \cdots)$. Then if the sets $V_{r}(x),(r=1,2, \cdots)$, satisfy conditions A, B, C of Alexandroff and Urysohn, the theorem is proved. Suppose that $V_{r}(a)$ and $V_{r}(b),(r>1)$, have a point in common and suppose $n_{r}(a) \leqq n_{r}(b)$. For convenience, let $p \equiv n_{r}(a)$ and $q \equiv n_{r}(b)$. Since the neighborhoods are monotonic decreasing, $U_{q}(b) \subset U_{p}(b)$. Then if $V_{r}(a) \equiv U_{p}(a)$ and $V_{r}(b) \equiv U_{q}(b)$ have a point in common, $U_{p}(a)$ and $U_{p}(b)$ have a point in common. But $p \equiv n_{r}(a) \equiv m\left[a, n_{r-1}(a)\right]$. Hence, by VIII, $V_{r}(b) \subset V_{r-1}(a)$. Likewise, if $n_{r}(a)>n_{r}(b)$, then $V_{r}(a) \subset V_{r-1}(b)$. Hence condition A of Alexandroff and Urysohn is satisfied.

Condition $\mathrm{B}$ is also satisfied. For two points $a$ and $c$, there is a $U_{n}(a)$ which does not contain $c$ since the logical product of $U_{1}(x), U_{2}(x), \cdots$ is $x$. By VIII, for any given $a$ and $n$, there exists an $m(a, n)>n$. For this $m$, no set $V_{m}(x)$ contains both $a$ and $c$. Suppose some $V_{m}(x)$ contains both $a$ and $c$. Since $U_{m}(x) \supset U_{n_{m}}(x)=V_{m}(x), U_{m}(x)$ contains both $a$ and $c$. Since $m>n, U_{m}(a) \subset U_{n}(a)$. Hence $U_{m}(x)$ and $U_{m}(a)$ have a point in common, namely $a$. Hence, by VIII, $U_{m}(x) \subset U_{n}(a)$. But $U_{m}(x)$ contains $a$ and $c$ and therefore $U_{n}(a)$ contains $a$ and $c$, which is a contradiction.

As for condition C, let $S_{r}(a)$ be the logical sum of all sets $V_{r}(x)$ which contain $a$. Since $S_{r}(a) \supset V_{r}(a)=U_{n_{r}}(a), S_{r}(a)$ is a neighborhood of $a$. Now for a given $a$ and $r$, by VIII, there exists an $m(a, r)$. For this $m, S_{m}(a) \subset U_{r}(a)$. For, let $b$ be any point of $S_{m}(a)$, that is, $b$ is any point of a set $V_{m}(x) \equiv U_{n}(x)$ which 
contains $a$. Now $U_{m}(x) \supset U_{n_{m}}(x)$. Hence $U_{m}(x)$ contains $a$ as well as $b$; also $U_{m}(a)$ contains $a$. By VIII then, $U_{m}(x) \subset U_{r}(a)$. Hence $b \subset U_{m}(x) \subset U_{r}(a)$ and $S_{m}(a) \subset U_{r}(a)$.

Since conditions A, B, C are satisfied, a distance function which satifies I, II, and III can be introduced directly in terms of the neighborhoods $\left\{V_{r}(x)\right\}$ by means of the notion of a chain of these neighborhoods joining two points $a$ and $b$.

This theorem gives conditions for the metrization of neighborhood spaces and a comparatively simple method of introducing the metric. It should be noted that condition VIII is a local condition in the sense that it is concerned with the neighborhoods of a single point $a$.

Condition VIII is not necessary for metrizability, but a necessary and sufficient condition can be given in terms of it.

THEOREM 4. A necessary and sufficient condition that a neighborhood space be homeomorphic to a metric space is that for every point $x$ there exists a sequence of neighborhoods, monotonic decreasing, and whose logical product is x, selected from the original neighborhoods and equivalent to them, satisfying VIII.

Proof. The preceding theorem proves the sufficiency of the condition. To prove the necessity, suppose the neighborhood space is metrizable. Then a distance satisfying I, II, and III may be introduced such that the spherical neighborhoods with this distance function are equivalent to the given $U$ neighborhoods. Then every $U$ neighborhood of $x$ contains a spherical neighborhood of $x$ and conversely. For each point $x$ of the space, select $U_{1}(x)$ such that the diameter of $U_{1}(x)$, namely $d\left[U_{1}(x)\right]$, is less than $1 / 2$, and select $U_{2}(x)$ so that $U_{2}(x) \subset U_{1}(x)$ and $d\left[U_{2}(x)\right]<1 / 4$, and in general $U_{n}(x)$ so that $U_{n}(x) \subset U_{n-1}(x)$ and $d\left[U_{n}(x)\right]<2^{-n}$. That the selection can be made in this way follows from the fact that the $U$ neighborhoods and these spherical neighborhoods are equivalent. The sequence $\left\{U_{n}(x)\right\}$ is obviously monotonic decreasing, its logical product is $x$, and it is equivalent to the original neighborhoods. To show that VIII is satisfied suppose a point $a$ and an integer $n$ are given. Then $U_{n}(a)$ contains a spherical neighborhood of $a, S(a)$. Call the radius of this spherical neighborhood $r$ and select $m$ so that $2^{1-m}<r$. Then this is the $m$ required by condition VIII. For suppose $U_{m}(a)$ and $U_{m}(b)$ have a point $x$ in common, and let $y$ be 
any point of $U_{m}(b)$. Then $a x<2^{-m}$ and $x y<2^{-m}$ from the condition on the diameters of $U_{m}(a)$ and $U_{m}(b)$. Hence we have $a y<2^{1-m}<r$, and therefore $y$ is in $S(a)$, which is contained in $U_{n}(a)$. Hence $U_{m}(b) \subset U_{n}(a)$.

The condition of this theorem has an advantage over the condition of Alexandroff and Urysohn. The latter condition postulates the existence of families of covering sets $\left\{G_{n}\right\}$ having certain properties, and in terms of these sets the distancefunction is defined. Given a neighborhood space, it might be difficult to determine whether such families of sets $\left\{G_{n}\right\}$ could be found. The condition of the present theorem also leads to the existence of such sets with the additional information that they are to be found among the original neighborhoods of the space.

The Pennsylvania State College

\section{AN INVOLUTORIAL LINE TRANSFORMATION DETERMINED BY A CONGRUENCE OF TWISTED CUBIC CURVES*}

BY J. M. CLARKSON

1. Introduction. Consider the pencils of quadric cones $K_{1}-\alpha K_{2}=0, L_{1}-\beta L_{2}=0$, each pencil having a common vertex which lies on all of the cones of the other pencil. For a given $\alpha, \beta$ the curve $C(\alpha, \beta)$ of intersection of the cones is composite, consisting of the line $l$ of the vertices of the two pencils and a twisted cubic curve $C_{3}(\alpha, \beta)$. As $\alpha, \beta$ take on all values independently, $C_{3}(\alpha, \beta)$ describes a congruence of space cubic curves. An arbitrary line $t$ of space will be bisecant to just one $C_{3}(\alpha, \beta)$, for any three points of space will determine a set of values for the parameters $\alpha, \beta, \rho$ in the system

$$
\left(K_{1}-\alpha K_{2}\right)-\rho\left(L_{1}-\beta L_{2}\right)=0
$$

of quadric surfaces, and if these three points be chosen on $t$, then $t$ lies on the quadric of (1) so determined and will meet $C_{3}(\alpha, \beta)$ twice. We shall henceforth write $C_{3}(t)$ for this curve.

Now consider a fixed plane $\pi$ and in this plane a Cremona involutorial transformation $\Gamma$ of order $n$ having a curve $\Delta_{m}$ of

* Presented to the Society, December 27, 1934. 